\title{
Synovial plica, lateral impingement and intra-articular impingement/entrapment pathologies of the temporomandibular joint
}

\author{
KenIchiro Murakami $^{1,2}$, Jeffrey J. Moses ${ }^{3,4,5}$, Howard Israel ${ }^{6}$, Joseph P. McCain ${ }^{7}$ \\ ${ }^{1}$ Department of Oral and Maxillofacial Surgery, Ako City Hospital, Ako, Japan; ${ }^{2}$ Kyoto University Postgraduate School of Medicine, Kyoto, Japan; \\ ${ }^{3}$ Pacific Clinical Research Foundation, Encinitas, CA, USA; ${ }^{4}$ Department of Oral and Maxillofacial Surgery, Loma Linda University, Loma Linda, \\ CA, USA; ${ }^{5}$ Division of Oral and Maxillofacial Surgery, School of Dentistry, University of California, Los Angeles, CA, USA; ${ }^{6}$ Touro College of \\ Dental Medicine at New York Medical College, Valhalla, NY, USA; ${ }^{7}$ Department of Oral and Maxillofacial Surgery, Massachusetts General Hospital \\ and Harvard School of Dental Medicine, Boston, MA, USA \\ Contributions: (I) Conception and design: K Murakami, H Israel, JJ Moses; (II) Administrative support: None; (III) Provision of study materials or \\ patients: None; (IV) Collection and assembly of data: All authors; (V) Data analysis and interpretation: All authors; (VI) Manuscript writing: All \\ authors; (VII) Final approval of manuscript: All authors. \\ Correspondence to: KenIchiro Murakami. Ex Associate Professor and Chief of Oral and Maxillofacial Surgery, Kyoto University Postgraduate School \\ of Medicine, Kitaku Ohmiya, Higashi-Onoboricho 37-3, Kyoto 603-8406, Japan. Email: murakeny@iCloud.com; keny_ako@yahoo.co.jp.
}

\begin{abstract}
Synovial plicae are common findings in most knees and extremity joints. However, plicae of the temporomandibular joint (TMJ) have not been adequately addressed. In the cadaver study, various plicae were observed with histologic findings in the research base, and clinically plica were detected in patients with internal derangement, recurrent luxation and other operative diseases in recent years. Although the incidence of a synovial plica varies between $24 \%$ with $62 \%$, the prevalence is unknown because of not detectable by MRI. Plicae in recurrent dislocation cases are relatively thick, wide and pliable with histology of dense fibrous/hyalinized tissues with scattered chondroid cells. In cases with internal derangement, plicae were thin pliable, and finger- or band-like with histology of fibrous tissues and/or inflamed synovium with vascular proliferation suggesting in pathogenesis. Although plicae may or may not be causing symptoms, when detected during surgery should be removed with other intraarticular pathologies for restoring the biological function of TMJ. In development of TMJ lateral impingement, the lateral capsular prolapse may play an important role. The soft tissues of the capsule become fibrotic and constricted with inflammation restricting mandibular movement. Early adhesive capsulitis appears as an adherent finger-like plica vascularized from the lateral capsule restricting movement of the attached disc. Plica lined with synovium combined with a deformed disc induce mechanical abrasion resulting synovitis, which may induce chondromalacia leading to deterioration of bone and joint. The release of adhesions along the lateral aspect of the capsule is performed, then lateral capsular stretching and repositioning of prolapsed synovial plica is favorable to obtain good outcomes. The term "entrapment" generally refers to a compression of a peripheral nerve or artery usually between ligamentous and bony structures. Whereas symptomatic synovial impingement and intra-articular synovial entrapment syndromes have been well documented in patient undergone total arthroplasty and joint replacements of the hip and knee. There is strong evidence that the TMJ is susceptible to the same symptomatic impingement and intra-articular synovial entrapment as other synovial joints. This review of our current state of knowledge will accelerate and update the evolution of further understanding of TMJ pathologies, diagnosis and treatment.
\end{abstract}

Keywords: Synovial plica; lateral impingement; synovial entrapment; temporomandibular joint (TMJ)

Received: 31 July 2020; Accepted: 16 September 2020; Published: 28 September 2020.

doi: $10.21037 /$ fomm-20-45

View this article at: http://dx.doi.org/10.21037/fomm-20-45 


\section{Introduction}

Temporomandibular joint (TMJ) synovial plicae, lateral impingement, and synovial entrapment following TMJ total joint replacement are intra-articular conditions with similar features. These conditions are likely related with a common etiology, and intra-articular pathologies can occur in pathologic TMJs as well as TMJs that have undergone total joint replacement surgery. Furthermore, the conditions and etiology of the group have commonality with other synovial joints.

Joint overload and the pathology and biochemical changes associated with it affect all of these intra-articular tissues. Ultimately synovial plica and impingement lead to synovial inflammation release of cytokines, resulting in chondromalacia and osteoarthritis (OA) based on the orthopedic models of the knee and other extremity joints. Whereas there are uncertain aspects in the model, there is a significant literature on TMJ pathogenesis that can help clarify the role of synovial plicae, impingement and synovial entrapment as an important cause of symptoms and biomechanical instability of the TMJ.

In combination the work of these authors on patients who have undergone arthroscopic and open TMJ surgery, along with their clinical research experience should enable us to present and discuss a way to forward to advance our knowledge of TMJ pathophysiology and provide a rationale for further evolution of clinical treatment.

\section{Synovial plicae and the TMJ}

Synovial plicae are common findings in synovial joints. Plicae are inward folds and excessive tissues of the synovial lining and are present in most knees and extremity joints. The literature describing synovial plicae indicates their origin comes from embryonic remnants of the septum of a joint cavity. In the knee joint, the synovial plicae are very frequent and often asymptomatic. The epidemiology of the prevalence of symptomatic plicae has not been clarified. The orthopedic literature indicates the usefulness of MRIs as a diagnostic modality and arthroscopy as a gold standard for diagnosis and as a treatment modality (1-3). An arthroscopically diagnosed synovial plica with evidence of inflammation and fibrosis, along with histologic confirmation is highly suggestive of a synovial plica with entrapment as a cause of symptoms (4). However, since symptomatic knee joints are likely to have additional pathologic tissues treated arthroscopically, it is difficult to analyze the responsibility of a synovial plica in determining the etiology of the patient's symptoms. It is believed that knee trauma, increased athletic activity and/or other factors cause tissue damage, synovitis, release of inflammatory mediators, ultimately leading to tissue fibrosis, decreased elasticity and localized chondromalacia involving the patella-femoral joint (5).

Since the TMJ is a synovial joint, one would expect the development of pathology and biological mechanisms involved with the formation of synovial plicae to be the same as other synovial joints. Although the presence of synovial plicae in cadaver TMJs haves been well documented in the literature $(6,7)$, the clinical study of plicae in TMJ disease has been first reported with the occurrence and a significant prevalence in recurrent dislocation and internal derangement observed in surgery cases (8). The study of arthroscopic and histologic findings in synovial membrane disease including plicae has recently reported (9). It remains to be determined if there is a correlation of arthroscopic pathology, biochemical changes in the tissues and synovial fluids of patients with symptomatic and asymptomatic plicae of the TMJ. Indirect evidence from studies of TMJ arthroscopy and synovial fluids suggest etiologic factors (e.g., joint overload, impaired mobility) which cause pathology in the cartilage and synovium, also affect TMJ synovial plicae.

\section{Embryology}

The formation of the human TMJ is from prenatal weeks of 8 to 10 . The establishment of mature morphologic pattern occurs at 12 weeks. The temporal bone is seen at 8 weeks as well, and the mandibular (glenoid) fossa and articular eminence are well formed by 22 weeks. Meckel's cartilage plays no part in the actual development of TMJ.

The attachment of the lateral pterygoid muscles is first noted at 8 weeks. At 10 weeks, the muscle is attached on the ball of condensing mesenchyme medial to the membrane bone of condyle. The articular disc formation is from weeks 12 to 14 . The inferior articular compartment is formed first by proliferation of blood vessels forming the inferior cleft. This is completed by 13 weeks, when the first cleavage is seen in the region that is to become the superior joint compartment. Innervation of the articular disc is seen early 12 weeks and completed by 14 weeks when both compartments are well organized. The mature morphologic shape of the articular disc is formed by 14 weeks with the development of a thin central region, increasing in thickness 
toward the periphery. The articular disc is composed of fibrous connective tissue and exhibits a high degree of vascularity except in the thin central region. In the perinatal stage of development of the TMJ, the synovium was clearly shown to be present in the lateral inner capsule of the superior compartment (10).

In the human TMJ, the inferior joint compartment is formed first, and followed by the formation of the upper joint compartment with the appearance of intervening connective tissue formation connecting to the lateral pterygoid muscle.

In most extremities, joints such as the knee, hip, and elbow, the origin of plicae are the remnants of the membranes that divide the synovial cavitations during normal joint development (2). On the contrary in the TMJ, the superior joint compartment is formed on the connective tissues of early articular disc and condyle. The common embryological origin of the synovial plicae from the developmental remnant of joint membrane is not adequately addressed in the human TMJ. Nevertheless, there is significance with the reporting that the plicae of the knee are anatomical structures not functionless embryonic remnants in the current anatomical study (11).

\section{Anatomy}

The human TMJ consists of superior and inferior compartments separated by an intervening articular disc, and with the hard tissue components of temporal and mandibular bone. The articular disc is firmly attaching to the lateral and medial pole of the mandibular condyle, and anteriorly connects with the lateral pterygoid muscle as well as posteriorly to retrodiscal connective tissue. The synovial membranes occupy the space between discal circumferences and inner capsule other than the bony articular surfaces. The upper joint compartment is larger than the lower joint space.

Major TMJ motion occurs in the superior joint compartment and the hinge motion is synchronously in the lower joint compartment. Thus, the human TMJ in natural mandibular movements are combined with translatory and rotatory joint motion.

It should be noted that external and internal rotations of the mandible are always synchronized in bilateral joint motions. During the common jaw motions of speaking, mastication and bite, tensile forth loading is continuously exerted to the lateral capsule and the articular disc of both TMJ.

\section{Anatomical dissection of the plicae in the cadaver (Figure 1)}

Murakami and Hoshino investigated the gross and arthroscopic regional anatomy, and microscopic histology of the TMJ in cadaver and described the details of the synovial plicae $(6,12)$. These plicae were observed as various types. Distinct tongue-like plicae (Figure $1 A$ ) were seen in the posterior synovial pouch as the protruding plica from the posterior synovial membrane in 11 of the 20 specimens $(55 \%)$. The average size of eight plicae measured was $5.0 \times 1.4 \mathrm{~mm}$ (6). Histologically, these tongue-like plicae were composed of a few superficial rows of cells and contained capillary blood vessels (12). In other anatomical dissections, wrinkle-like synovial folds were detected in the anteromedial aspect of upper joint space in specimens with anterior disc displacement (Figure 1F). Ohmura reported on the study of the gross anatomy and histology of synovial plicae found on the lateral capsular inner wall of the TMJ. He had reported the meniscus-like, crescent-shaped plica with their bases on the inner aspect of the lateral wall of the superior joint compartment with histologic findings of synovium and sub-synovial tissues (13).

\section{Surgical and arthroscopic view of plicae in patients series}

A description of synovial plicae can be found in the TMJ surgery literature $(8,9,14-17)$. Moses (14) stated the presence of the synovial plicae in stage 2 of TMJ lateral impingement together with chronic adhesive capsulitis, proliferative synovitis, discal displacement or immobility. Kirk $(15,16)$ classified the coronal plane pathology-impingement to type I to III based on surgical and MRI findings, and described the occurrence of "plica" with biomechanical considerations. He also stated that large destabilizing plicae might be seen in lateral impingement cases, and that it was necessary to release the plica along with the disc from the lateral aspect of the fossa and articular tubercle (17).

Murakami et al. reported the occurrence of synovial plicae in a case series of TMJ open surgery (8). The surgical appearance, distribution, and histologic findings of plicae were described among 20 patients with TMJ recurrent dislocation and internal derangement. The incidence of the synovial plica was three of four joints with internal derangement, and 15 of 24 joints $(62.5 \%)$ with recurrent dislocation. Observed synovial plicae in patients with dislocation group (Figure 2) were various forms and mostly broader than those of the internal derangement group. Forms were meniscus-like plica (Figure $2 A$ ), broad/wide 

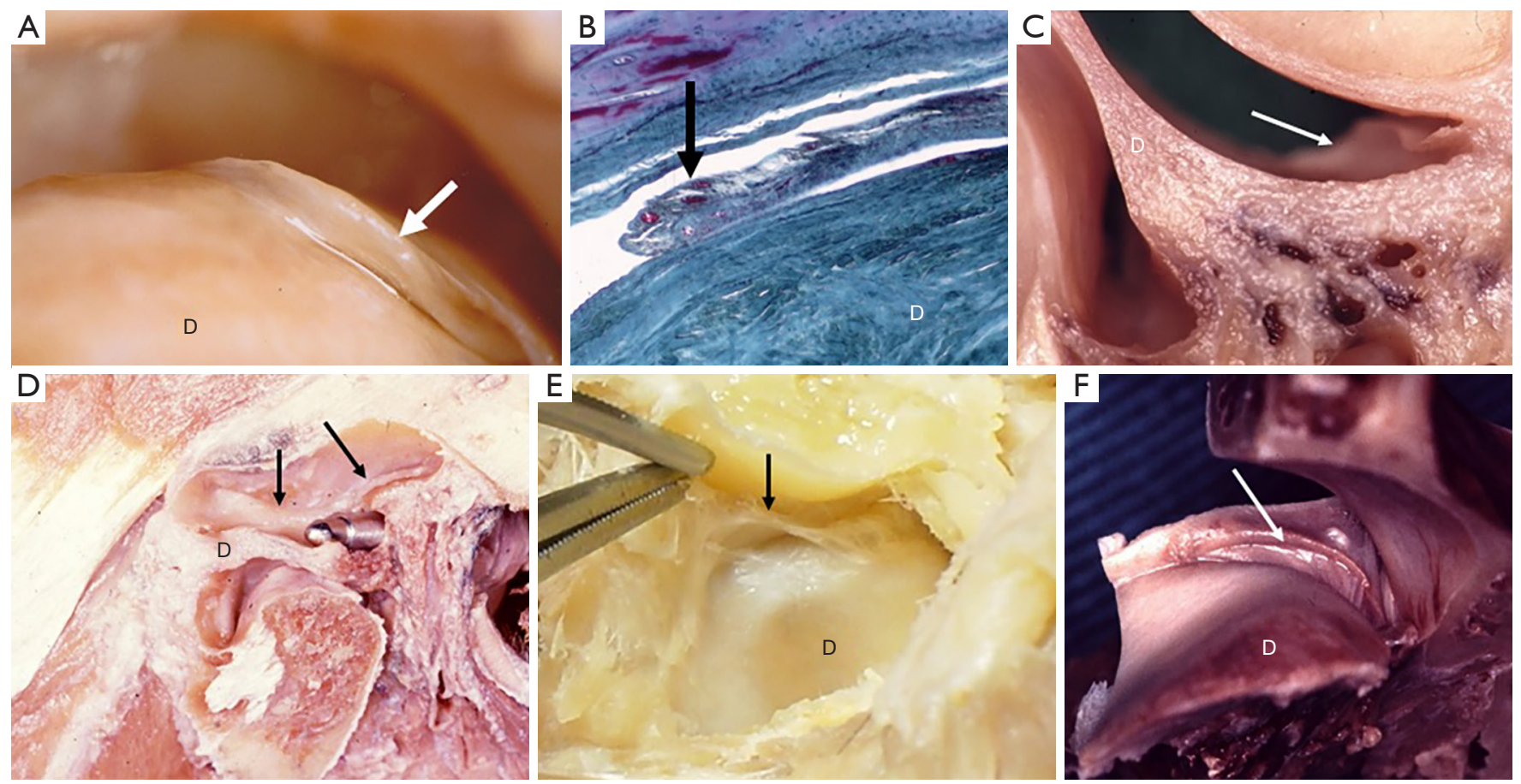

Figure 1 Various forms of plicae in the cadavers (small D: articular disc, arrow: plica in the photo). Upper left (A): tongue-like plica; upper middle (B): histology of a tongue like plica composed of a few superficial rows of cells and contained capillary blood vessel (Goldner's trichrome stain, low power magnification); upper right (C): fringe like plica. Lower photos: band like plicae; lower left (D): plica at inner lateral wall (demonstrate of arthroscopic puncture by small blunt trocar); lower middle (E): plica at lateral groove; lower right (F): winkle like plica at anteromedial recess in the disc displacement specimen. (Murakami K, 2020, unpublished data).

(Figure $2 B$ ), or tongue-like (Figure $2 C$ ) in shape, and with arthroscopic view of tongue-like (Figure $2 D$ ) and rounded form of plicae (Figure 2E). These plicae were detected as relatively thick, wide, pliable areolar tissue lying within the upper posterior pouch protruding onto the disc, or seen at the lateral and medial synovial grooves.

The particular patient with internal derangement of closed lock suspecting of early lateral impingement, the plica was detected at lateral synovial groove with a tight lateral inner synovial wall of the capsule with jaw opening (Figure $3 A, B)$. The apparent band like plica at the medial inner wall of the capsule was also documented by McCain (Figure 3C,D). A recent study by Hakim et al. (9) correlated specific arthroscopic findings and histologic results from arthroscopic guided synovial biopsies in 210 patients with an arthrogenous TMJ disorders with painful jaw movements and limited opening, and stated that the synovial plicae (finger-like projections from the synovium) were detected in $24.8 \%$ of the observed joints in 210 patients.

\section{Histopathologic finding of plica in patient series (Figures 4,5)}

In the patients with recurrent dislocation (8), the most consistent finding (12 of 14 specimens) was dense fibrous tissues with scattered chondroid cells (Figure 4A,B), and hyalinized tissue with clusters of chondroid cells (Figure $4 C, D$ ). Distinct cartilaginous tissue was present in 2 of 14 specimens, and calcification was detected in 1 of 14 specimens of synovial plica in this study (Figure 4E). Cartilaginous tissue and calcification appeared only in the dislocation group, while the synovial cells at the lining tissues were also observed in certain specimens. In the particular patient with internal derangement, there were interesting histologic findings of plicae consisting with fibrous tissues with mildly inflamed synovium (Figure 5).

Hakim et al. disclosed that there was a statistically significant association of petechiae synovitis with lymphoplasmacytic infiltrate, petechiae synovitis and adhesions with hyperplastic synovium, joint stenosis with chronic inflammation, pannus formation with fibroblast 

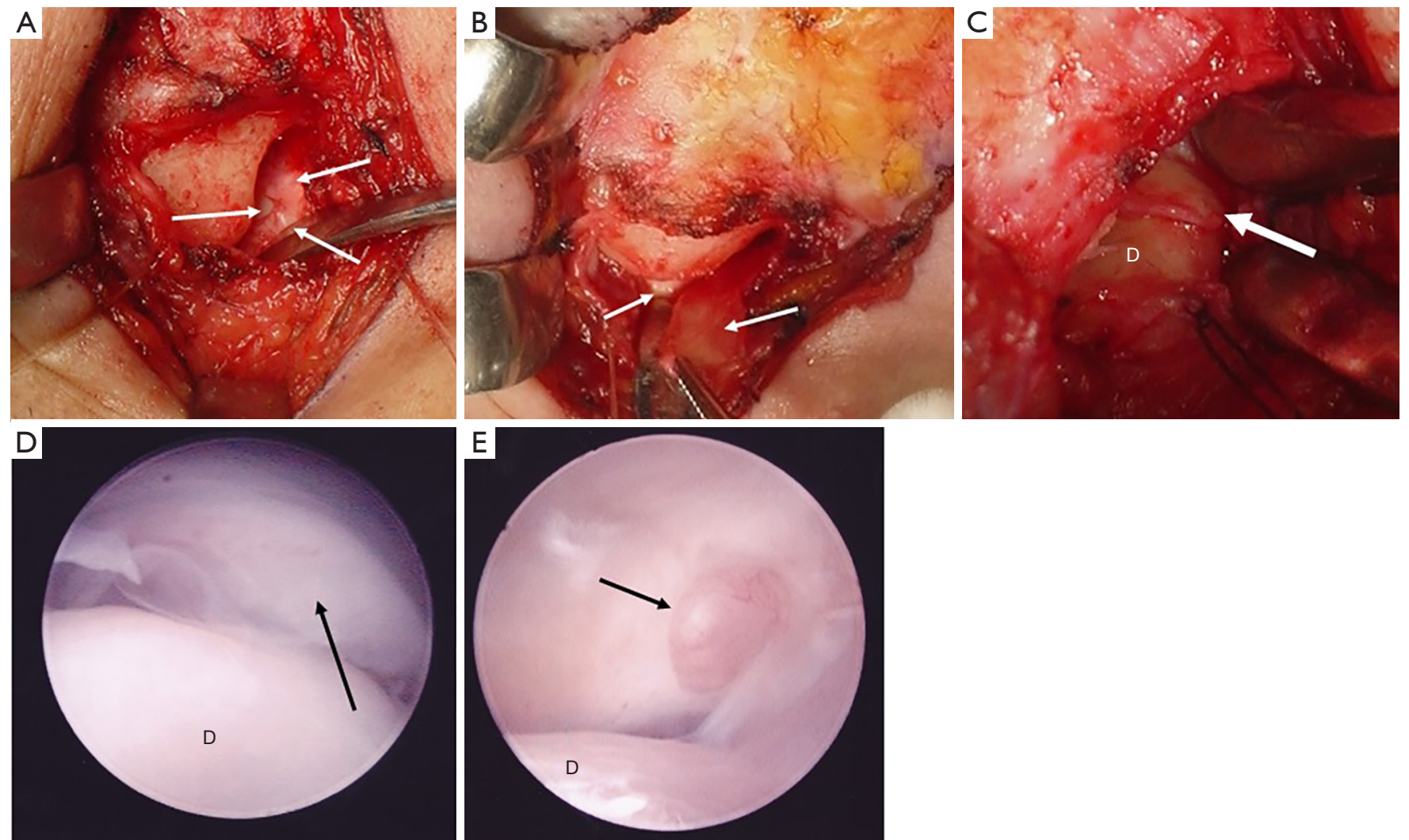

Figure 2 Surgical and arthroscopic views of plicae in patients with recurrent luxation (D: articular disc, arrow: plica). Upper left (A): meniscus like plica; upper middle (B): broad form; upper right (C): tong like plica; lower left (D) and right (E): arthroscopic view of tongue like plica protruding from the posterior pouch and rounded form of plica in the medial groove. (Murakami K, 2020, unpublished data).

proliferation, and synovial plica and joint stenosis with vascular proliferation (9).

\section{Discussion and prospective}

Various forms of synovial plicae were detected in TMJ cadaver (6) and surgical patients with recurrent dislocation and internal derangement (8). The reason why the author (KM) remarked and observed the plicae in the previous cadaver study was from the description of plica synovialis mediopatellaris of knee joint in the Atlas of Arthroscopy (18), in where state it causes joint internal derangement only when it is too big or become too hard with figures. Although the similarity of TMJ plicae to knee joint plicae was suspected, the role of synovial plica in creating sign and symptoms in TMJ disorders remains uncertain.

In the recurrent dislocation group, the form of plicae were broad and meniscus-like, or tongue-like with relatively thick and pliable. The histological findings revealed fibrosis and cartilaginous tissue. These findings suggest that plicae seems to be involved with joint inner space occupation, maintaining the intraarticular space to compensate for enlargement of volume due to hypermobility. On the contrary, a finding of calcification in the cartilaginous tissue of the plica may suggest the sequences of frequent intraarticular mechanical interference between plica and articular surface. This may relate to the possible risk factor of progression to long standing dislocation due to heavy friction/abrasion between plica and the disc/eminence. Actually, immediately after removal of the plica during surgery, the articulation of the condyle with disc toward to the eminence became smooth frequently. Therefore, the removal of synovial plicae might be indicated in patients with open lock phenomenon, jaw hypermobility and recurrent luxation.

In contrast, the histological finding of plica in patients with Wilkes stage IV internal derangement showed synovial 

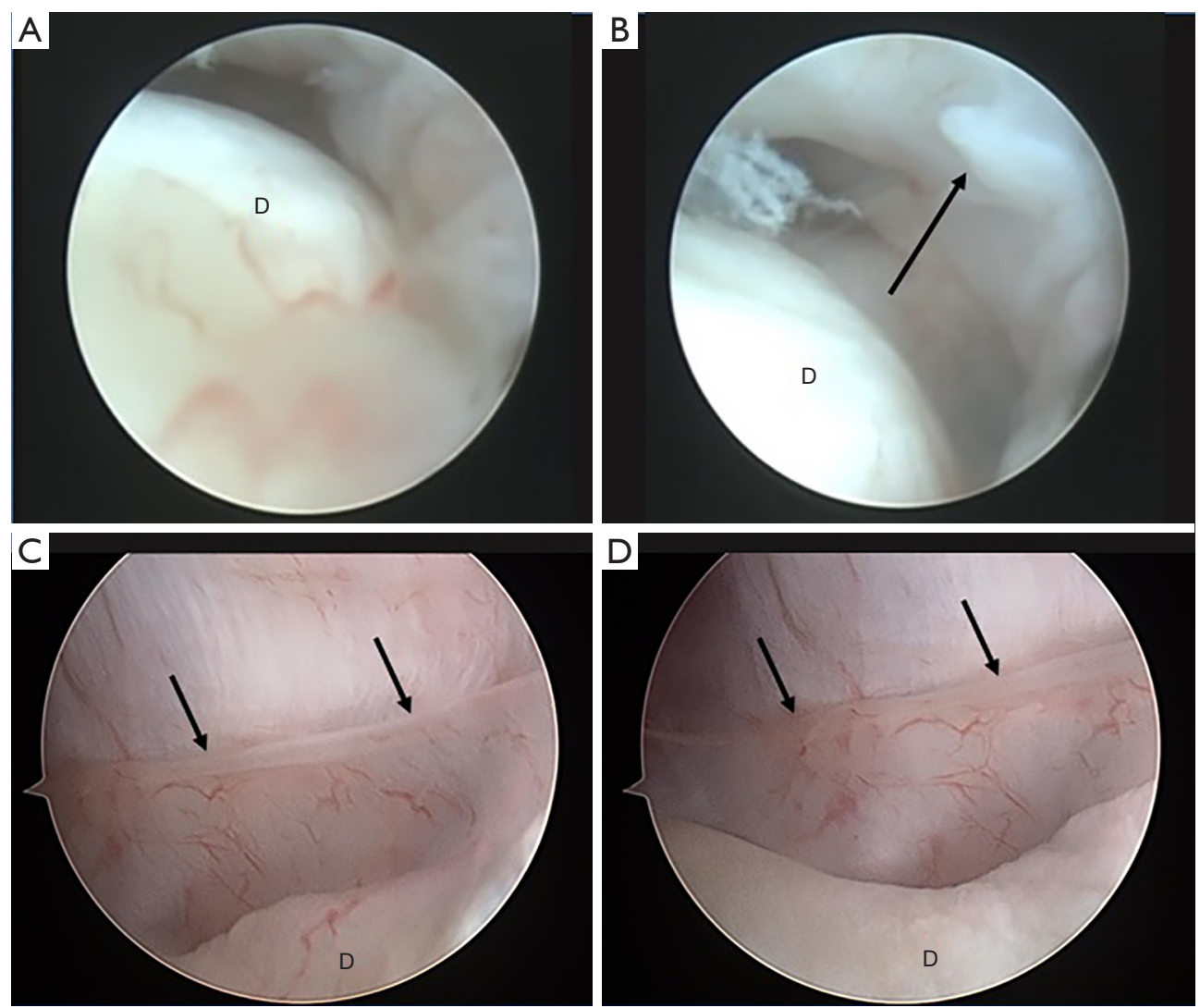

Figure 3 Arthroscopic view of plicae in patient with internal derangement (D: articular disc, arrow: plica). Upper left (A): view of upper joint space with lateral aspect from the posterior in closed jaw; upper right (B): same TMJ in jaw opening view. (Murakami, 2020, unpublished data); lower left (C) and right (D): synovial band like plica at the medial inner wall of the capsule. (McCain JP, 2020, unpublished data).

inflammation that was observed on the upper part of the synovial plica. In the surgery/arthroscopy for almost stage 4 and 5 internal derangement cases, there is observed middle to severe synovitis on the posterior attachment. This suggests the possibility of pathogenic role of synovial plica associated with synovitis in painful internal derangement.

An important arthroscopic study of symptomatic pathologic mediopatellar plica and asymptomatic nonpathologic suprapatellar plica of the knee compared substance $P$ and nerve fiber density of plica tissue specimens (19). There were significantly higher levels of substance $\mathrm{P}$ and nerve fiber density in mediopatellar plica as compared with asymptomatic suprapatellar plica specimens in these patients. The symptomatic mediopatellar plica also demonstrated pathologic changes that were present arthroscopically. The authors concluded that substance $\mathrm{P}$ expression in symptomatic plica may be the important pathophysiological basis for pain.
Since the mid 1990s, TMJ synovial fluid analysis has been investigated by Kopp and his group; the neuropeptides substance $\mathrm{P}$, calcitonin gene-related peptide, and neuropeptide $\mathrm{Y}$ have all been found at high levels in the synovial fluid of arthritic TMJs and IL- $1 \beta$, TNF- $\alpha$, prostaglandin E2 (PGE2) and leukotriene B4 as well (20). Yoshida et al. (21) showed the immunohistological expression of substance $\mathrm{P}$ in TMJ surgery specimens particularly intense at the margin of the TMJ disc and synovial membrane layer, on the surface of hypertrophic synovium and around the newly formed capillaries in the TMJ discs. They discussed the clinical symptoms of internal derangement of the TMJ were thought to be associated with the degree of synovitis. Sato et al. reported the unique immunohistochemical study that the extent score of cells stained for substance $P$ in joints with internal derangement was significantly higher than the controls. The extent score of these cells, however, did not correlate with pain 

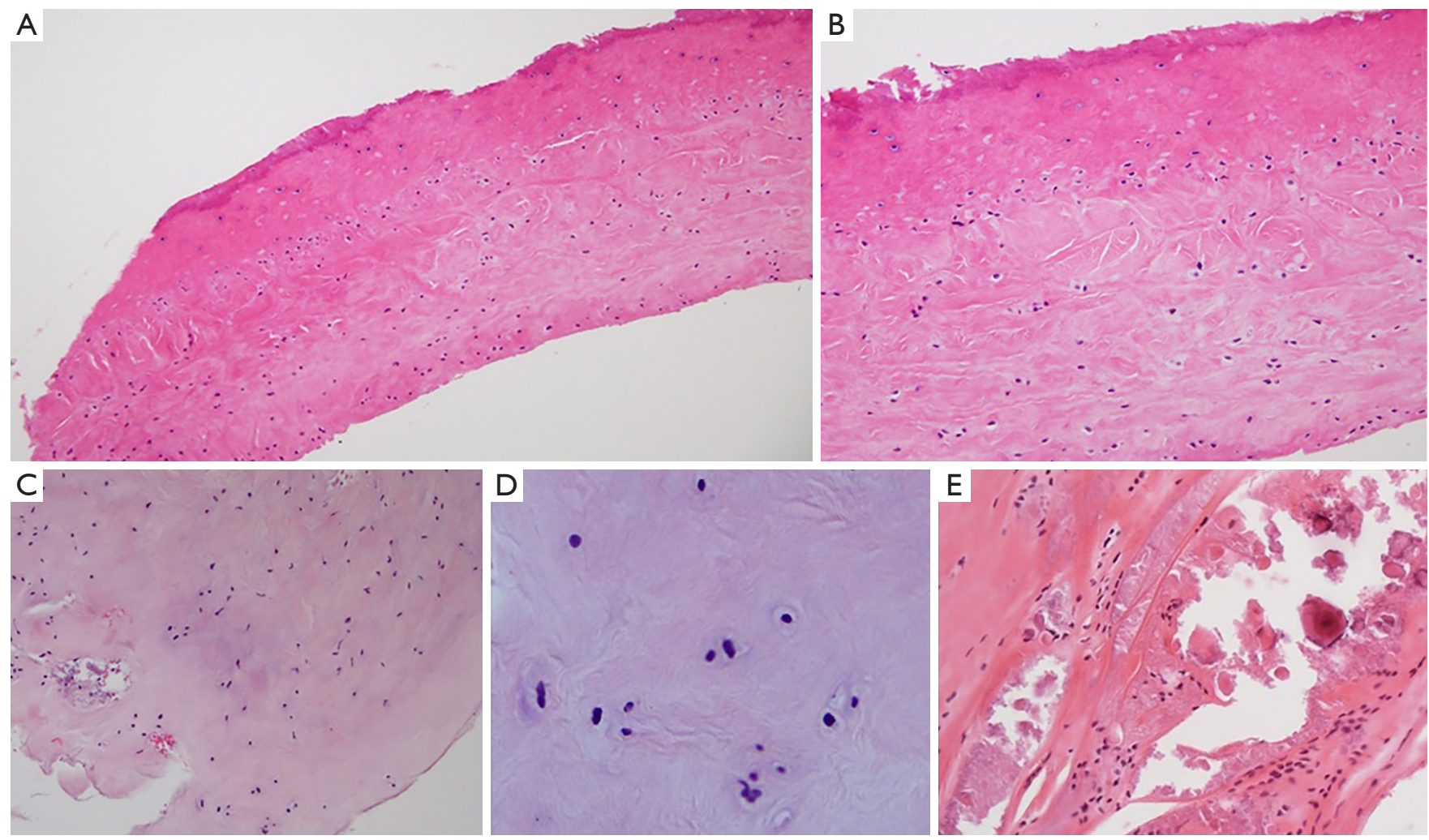

Figure 4 Surgical pathology of plicae in patients with recurrent luxation. Upper left (A): fibrous tissue (H-E stain, 100×); upper right (B): dense fibrous with scattered chondroid cell (H-E stain, 200x); lower left (C): hyalinized tissue with chondroid cells (H-E stain, 200x); lower middle (D): cluster of chondroid cells with extracellular matrix (H-E stain, 400x); lower right (E): fibrous tissue with part of calcifications (H-E stain, 200×). (Murakami K, 2020, unpublished data).
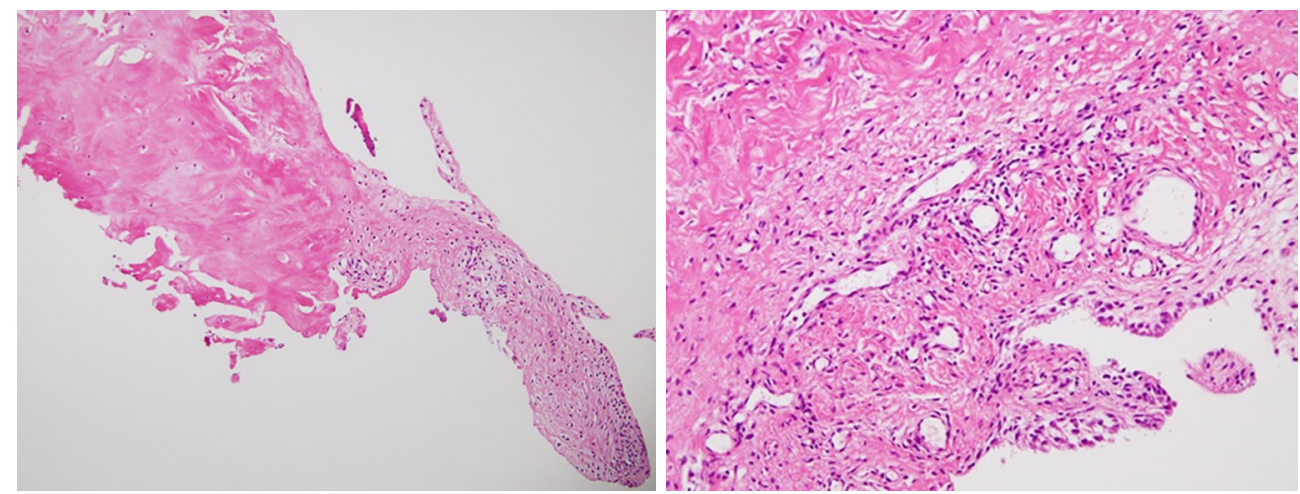

Figure 5 Surgical pathology of plica in patient with internal derangement. Left: part of fibrous tissue is transferring to synovial inflammation (H-E stain, 100x); right: part of inflammatory synovial tissue (H-E stain, 200×).

in the joint or the degree of synovitis (22). However, the neuropeptide include substance $\mathrm{P}$ in neurogenic inflammation and the release of pro-inflammatory cytokines are likely to play an important role in the pathologic tissue changes that can be visualized arthroscopically in inflamed and fibrous synovial plica of TMJ, although this requires 
further investigation.

In the study of the matrix metalloproteinases (MMPs) and tissue inhibitors of metalloproteinases (TIMPs) in knee OA progression, Yang et al. clearly showed and discussed that the mechanical abrasion of the medial femoral condyle by the medial plica during knee motion causes inflammation in the plica. IL-1 $\beta$ and TNF- $\alpha$ were detected in plica as well as in pannus-like tissues of knee OA patients. These two cytokines upregulate MMPs and TIMPs, resulting in a TIMP/MMP imbalance, which may lead to the destruction of articular cartilage (23). This model may apply to the progression of the pathologic sequences in TMJ internal derangement. Overloading and tensile stresses to the anterior synovium or to the posterior attachment as well as to medial and lateral inner synovial capsular wall may causes synovial folding followed by plica formation. Plicae lined with synovium combined with a deformed disc induce mechanical abrasion resulting inflammation in the synovial membranes and intraarticular plicae, which may induce chondromalacia leading to deterioration of bone and joint. Previous arthroscopic studies have clearly shown an accumulation of data that synovitis, adhesions, and chondromalacia were the main causes of intra-articular pathogenesis leading to Wilkes stage 3 to 5 internal derangement (24-26). Also, TMJ synovial fluid analysis has clearly indicated that the increased level of pro-inflammatory cytokines including IL- $1 \beta$ and TNF- $\alpha$ related to upregulation of MMPs and TIMPs resulting degenerative changes (26-28).

In the current study of TMJ arthroscopic biopsy with histology of the synovial diseases, relationships between synovial plicae and joint stenosis with vascular proliferation as well as synovial hyperemia and disc displacement with degenerative changes were reported (9). By the latest immunohistochemical study for TMJ, the densitometric expression of MMP-7 and MMP-9 was significantly increased in the synovium in patients with internal derangement than in the control (29). These results demonstrate the need for further studies to determine if synovial plicae demonstrate similar immunohistochemical findings in patients with TMJ inflammatory/degenerative diseases.

There are limited data regarding synovial plica in TMJ, exemplified by the few reports on the incidence of synovial plicae. In orthopedics, the MRI is useful to detect the presence of plica and arthroscopy is the gold standard for the diagnosis [1-5]. But in TMJ plicae, routine 1.5 Tesla MRI is insufficient to detect intra-articular detail of structures (30) such as plicae. The plica may or may not be major cause of symptoms. The synovial lining throughout the joint (particularly the posterior attachment) and its inflammation (synovitis) seems to be essential for biochemical pathways leading to progression to inflammatory/degenerative TMJ disease. Arthroscopy and the histological study of plicae haves just started. To further the development of our knowledge of the pathophysiology of TMJ disorders, when plicae are detected under arthroscopy and/or open surgery, it should be removed and submitted for histopathological examination as well as immunohistochemical study.

\section{Biomechanical adversity and joint load distribution}

It has been postulated that biomechanical adversity leading to joint force load and shear concentration within the TMJ such as those generated, for example by skeletal apertognathia and high mandibular angle orthometrics, this may overcome the physiologic joint lubrication (31) and debris phagocytosis mechanism references earlier mentioned in this manuscript. Orthopedic biomechanical literature generally supports the concept that joint health is dependent upon joint load distribution rather than load concentrations and shear force generation. In the treatment of TMJ internal derangement, a shift from disc position paradigm to disc mobility and biomechanical joint adaptive enhancement potential via production of disc mobility and removal of translatory impedance was published (32). Its philosophy based upon the early work entitled the effect of arthroscopic surgical lysis and lavage of the superior joint space on TMJ disc position and mobility describing MRI findings of disc position and mobility (33). It was found that the mobility of the disc postoperatively as presenting on the preoperative and postoperative MRI scan correlated best with the reduction of pain and the increase of jaw function mobility (no matter what Wilkes stage was present) (Figures 6,7).

The further study revealed patient success from arthroscopic lysis, lavage, capsular stretch and eminence release procedures documented in a cohort of 237 patients studied over a 2 -year period having a success rate of over $92 \%$ for pain reduction and increased range of motion (31). This work was followed for an initial period of 2 years with $92 \%$ and then for over 10 years with a maintenance of over $90 \%$ clinical success in pain reduction and range of motion according to the original criteria of 

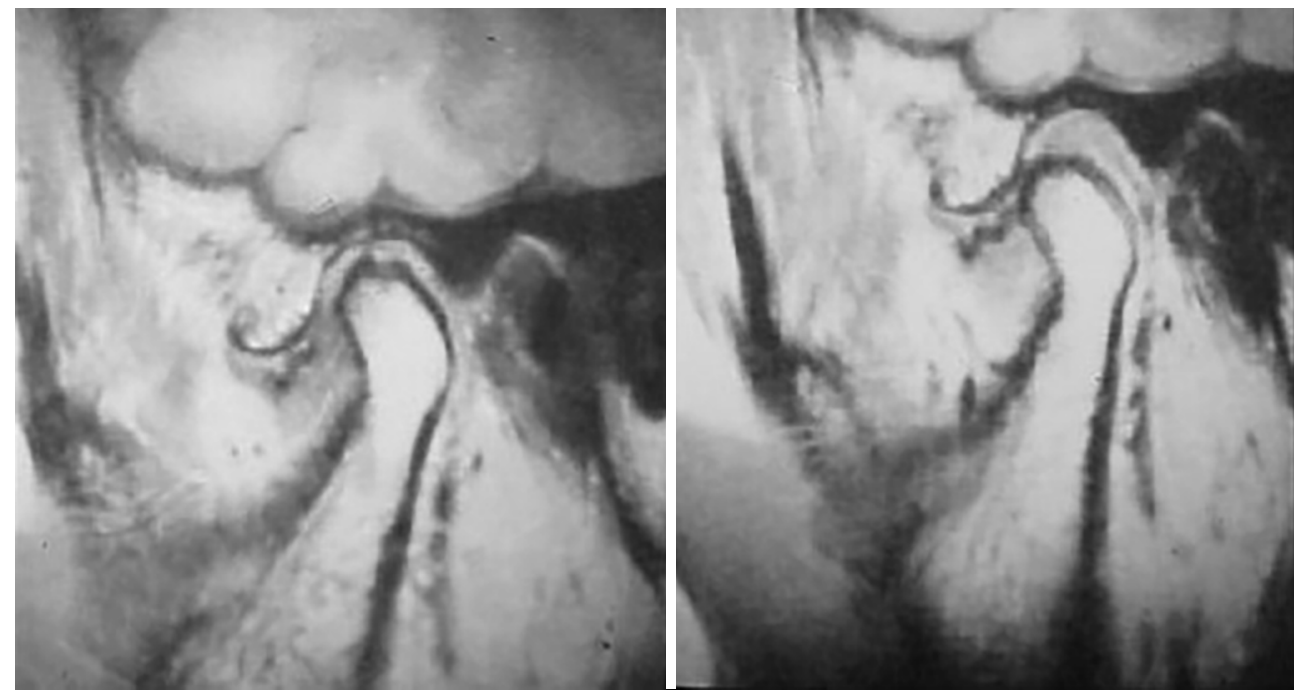

Figure 6 Preoperative disc immobility case. Reprinted with permission from reference (33).
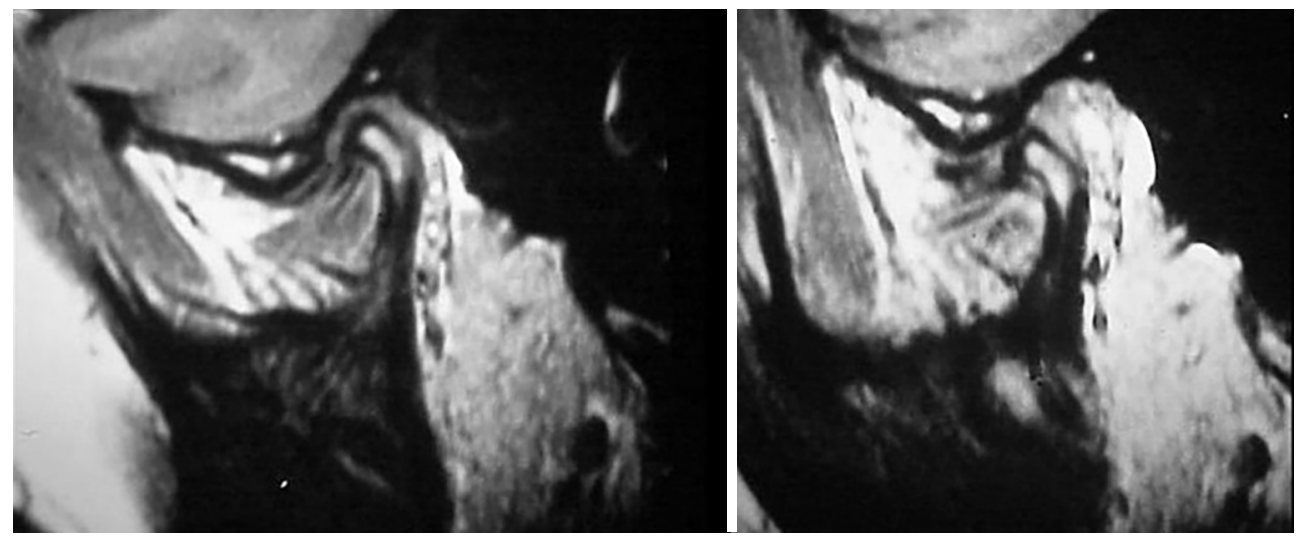

Figure 7 Postoperative disc mobility obtained case. Reprinted with permission from reference (33).

the study when combined with the patient having their diagnosed biomechanical adversity addressed sequentially following their arthroscopic procedure. For example only, patients with high angle skeletal-occlusal apertognathia undergoing a bimaxillary orthognathic surgical procedure would have this scheduled after their re-establishment of disc mobility, physiologic restoration of load distribution with physical therapy and adaptive orthotic management being applied until the performance of their final orthognathic surgery (31). With increasing the capacity of cells to have mesenchymal reprogramming, remodeling would occur within the limits of biomechanical function, but reduction of pain with restoration of jaw function would be the result.
Interestingly, and requiring further study, remodeling of the articular surfaces was noted on follow-up tomography and a follow-up study was published (34) (Figure 8). This study showed that there was a direct correlation of the cases with having articular remodeling to the patients having symptoms greater than 6 months preoperatively, but this was irrespective of clinical pain reduction and increased ROM success which was ascertained to have been maintained in this study.

\section{Lateral impingement}

Moses (14) described and stated firstly the lateral impingement syndrome in TMJ, in where 3 stages of the 

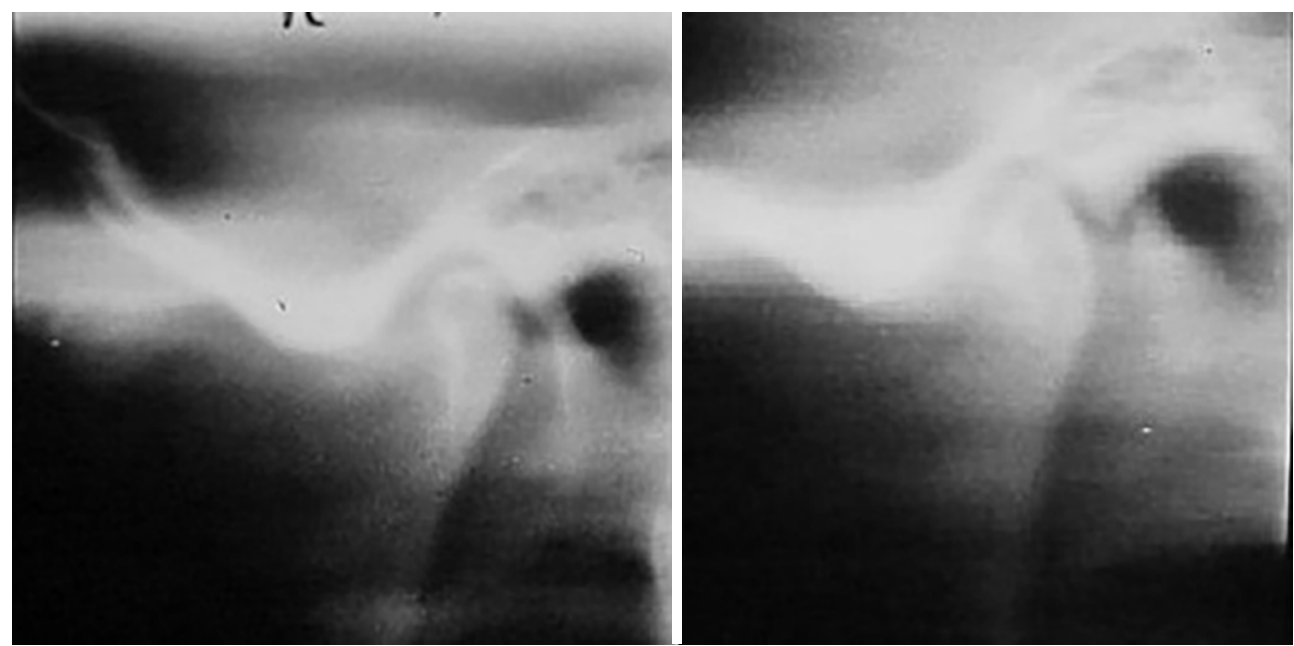

Figure 8 Adaptive remodeling: preoperative (left) and postoperative 1-year tomography (right). Reprinted with permission from reference (14).

Table 1 Progressive stages of impingement lesions

\begin{tabular}{llll}
\hline Stage & Diagnosis & Clinical course & Treatment \\
\hline I, inflammation & Acute capsulitis and synovitis & Reversible & $\begin{array}{l}\text { NSAID, rest, physical therapy, orthotic splint, } \\
\text { intracapsular irrigations and lavage }\end{array}$ \\
II, fibrosis & $\begin{array}{l}\text { Chronic adhesive capsulitis, proliferative } \\
\text { synovitis, discal displacement or immobility, } \\
\text { synovial plicae }\end{array}$ & $\begin{array}{l}\text { Recurrent pain } \\
\text { with activity }\end{array}$ & $\begin{array}{l}\text { Arthroscopic lysis of adhesions, lateral } \\
\text { eminencia release and capsular stretch, lavage, } \\
\text { physical therapy, OSA }\end{array}$ \\
$\begin{array}{l}\text { III, bony remodeling } \\
\text { and attachment } \\
\text { migration }\end{array}$ & $\begin{array}{l}\text { Discal displacement or immobility, hyperplastic } \\
\text { eminencia tubercle, degenerative joint disease }\end{array}$ & $\begin{array}{l}\text { Progressive } \\
\text { disability }\end{array}$ & $\begin{array}{l}\text { Lateral eminencia release, capsular stretch, } \\
\text { lateral eminencia osteoplasty, physical therapy, } \\
\text { OSA }\end{array}$ \\
\hline
\end{tabular}

Reprinted with permission from reference (14). OSA, orthotic splint appliance.

impingement lesion with diagnosis, clinical courses and treatment were presented (Table 1).

On attempted opening, lateral adhesive components can cause in-coordination of disc/condyle/eminence dynamics. Strain is placed on the lateral disc attachments as the condyle is forced to begin translation from within the inferior joint compartment. The disc, while relatively immobile in its relationship to the articular eminence at the lateral capsular region due to adhesive capsulitis, may or may not be displaced at this time.

Over a period of time, translation solely in the inferior joint compartment may cause a gradually increasing laxity of the lateral disc attachment, allowing the anatomic migration of the disc anteromedially. As the disc slowly migrates forward and medially, movement within the superior joint compartment generally remains minimal.
The inferior joint compartment begins to act as the translatory compartment for the "wide-open" mouth position. Anterior disc displacement traditionally has been diagnosed via arthrographic studies, and more recently with MRI. A sagittal view may inaccurately reveal a wellplaced disc; however, the MRI coronal view more accurately allows diagnosis of medial or lateral disc displacement. MRI correlative studies on more than a hundred arthroscopic confirmations of disc displacement have shown that discs which appeared in normal anatomic position on sagittal views were actually rotated on their condyles with medial displacement (Figure 9).

Diagnosis of disc pathologies, based on two-dimensional studies, can be misleading. In some cases, the posterior band almost becomes longitudinally placed antero-posteriorly along the lateral rim of the condyle. This may lead to 

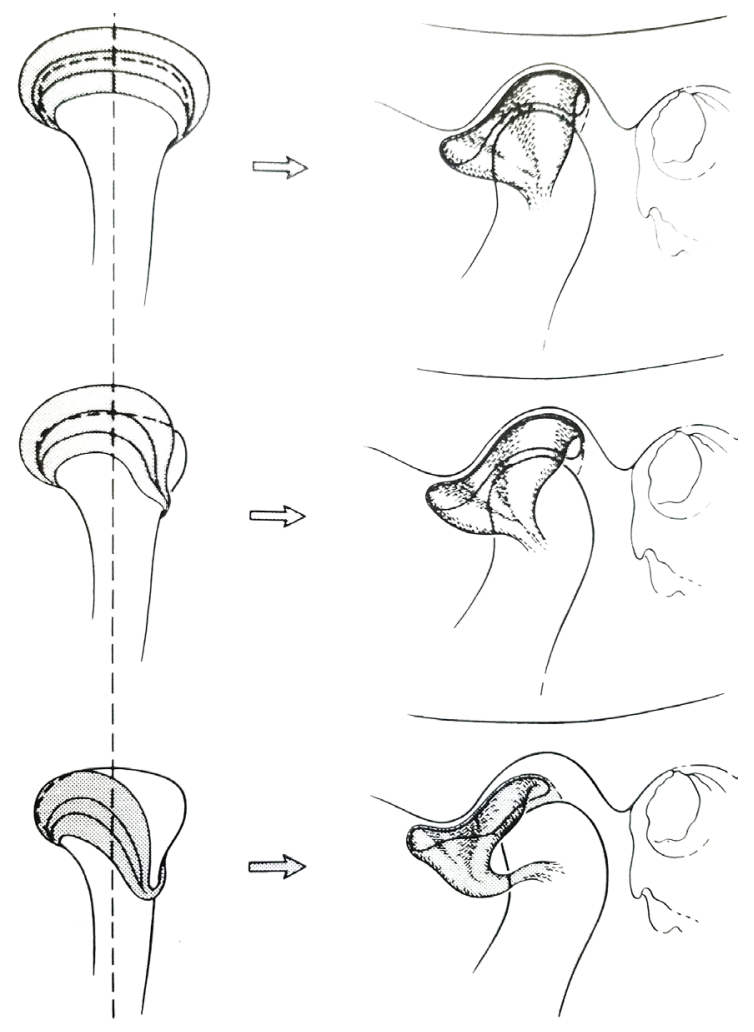

Figure 9 Disc appears well placed on both coronal (left) and sagittal imaging (right) at the top. Disc rotated on the condyle with medial displacement in the middle schema. Consequently, disc increases the laxity of the lateral collateral discal attachment to the condyle. Reprinted with permission from reference (32).

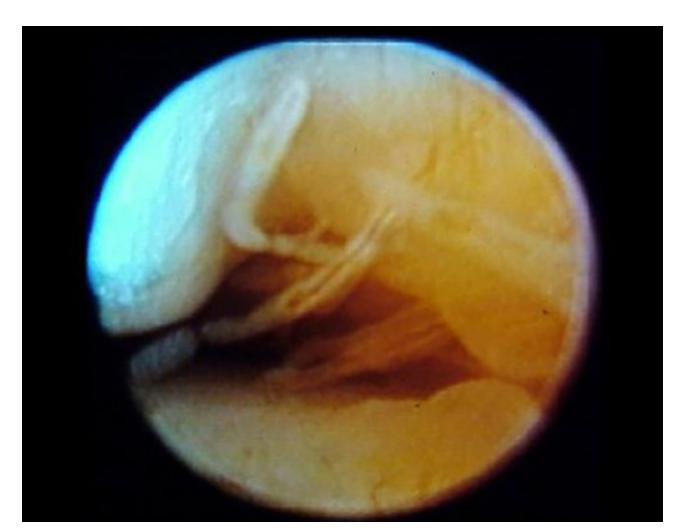

Figure 10 Finger-like plicae vascularized from the lateral capsule restricting movement of the lateral superior portion of the disc. Reusing from reference (35).

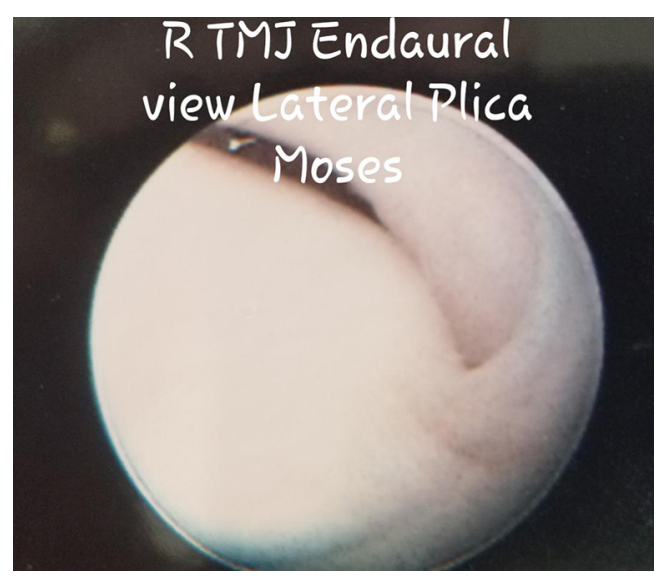

Figure 11 Lateral capsular prolapse and plica viewed by endaural arthroscopic view. (Moses JJ, 2020, unpublished data).

a "bulging" out of the capsule on coronal MRI. Early adhesive capsulitis appears as an adherent finger-like plicae vascularized from the lateral capsule restricting movement of the lateral superior portion of the disc (Figure 10).

In other cases, coronal MRI images of a disc more medially displaced may have a "sucked-in" appearance of the lateral capsule on coronal MRI, which is termed lateral capsular prolapse or plica when viewed via endaural view arthroscopy (Figures 11,12). Lateral capsular prolapse may play a role in the development of the lateral impingement phenomenon. A review of arthroscopic cases reveals that most pathologic adhesions and restricted motion lie within the lateral third of the joint. The soft tissues of the capsule become fibrotic and constricted, with inactivity and/or inflammation restricting mandibular movement. From the endaural arthroscopic approach, viewing anteriorly along the lateral synovial groove of the superior compartment a particular patient showed inflamed synovial proliferation and projections (Figure 13) as well as adhesions binding the disc to the eminence and capsule, leading to restricted mobility and possibly pain.

The background for the development of the arthroscopic treatment methods for the lateral impingements was his arthroscopy experience, particularly by his endaural portal observations (36) and the touching to a concept of the acromial impingement syndrome in shoulder. He described that review of studies similar joint pain 

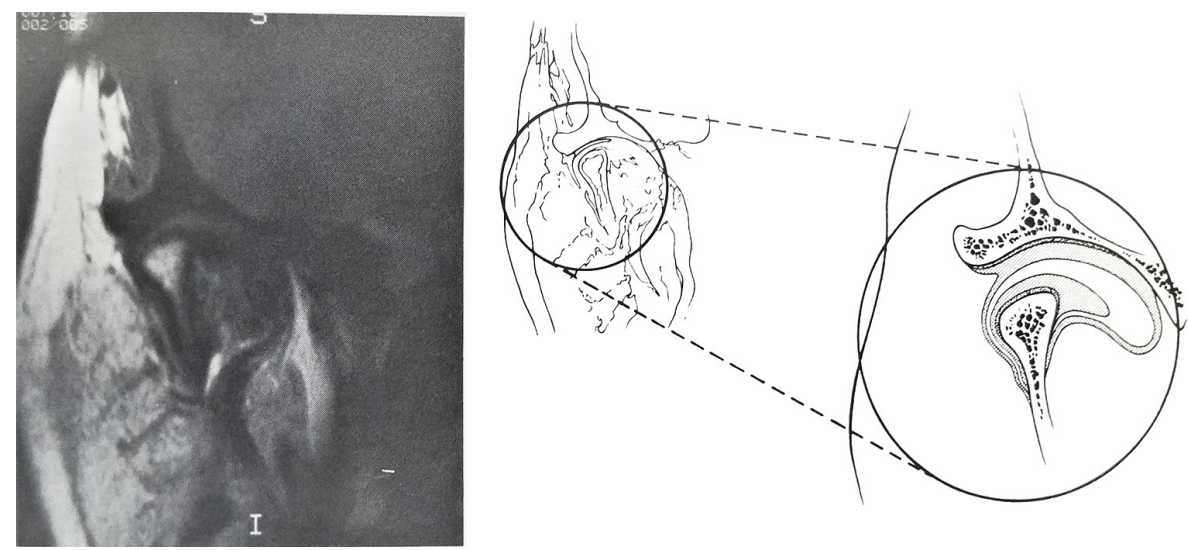

Figure 12 MRI coronal view (left) and drawing (right) shows the lateral capsular concavity and medial disc displacement. Reprinted with permission from reference (14).

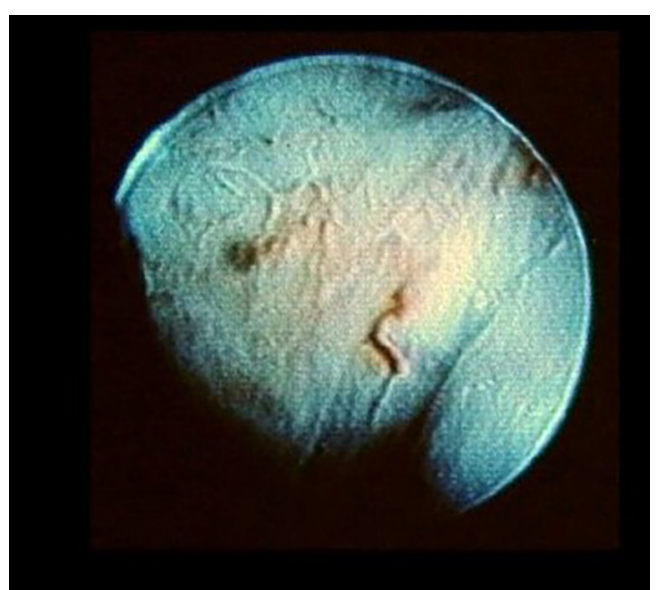

Figure 13 Inflamed synovial proliferative tissue and early adhesions. (Moses JJ, 2020, unpublished data).

and restricted mobility in the shoulder with acromial impingement syndrome shows a striking resemblance to observations made on the TMJ (14). Thereafter the observed intraarticular pathology including plicae were documented and he developed and a unique surgical intervention of lateral eminencia release and capsular stretch with conventional arthroscopic lysis and lavage (37). By his reflective story, this procedure of "lateral eminence release and capsular stretching" of the fibrotic capsule was inspired through conversations with his friends in the orthopedic community who suggested that with repetitive injury and inflammation, there was fibrosis and shortening of the lateral retinaculum "ligament" of the knee and they would perform a "lateral release" which would allow the retinaculum ("ligament") to be stretched or to be separated from their attachments to the bone and re-attach at a different level thereby allowing more patellar freedom of movement without pain and restriction. Moses took this principle to the vertical and transverse lateral TMJ ligaments (Moses J, personal communications, 2020).

Regarding the plica in surgery, the release of adhesions along the lateral aspect of the capsule is performed, then lateral capsular stretching and repositioning of prolapsed synovial plicae was carried out. Moses described that adhesions were lysed with a variety of methods ranging from indirect release with a blunt trocar to a combination of sharp and blunt dissection using a triangulation technique under optically assisted direct vision (35) and reported the good outcome (36). Moses's recent concept of TMJ surgery is that the plica itself does not need be excised, but rather the surgeon performing a procedure to free up the superior aspect of the lateral capsule attachment as well as the insertion of the oblique and vertical TMJ lateral ligament to its corresponding lateral eminence bone would be beneficial in obtaining freedom of motion without discomfort. Also he stated that performance of a lateral plica excision, even with a HO-YAG laser is potentially dangerous for perforation and damage to the facial nerve outside of the capsule and access through the use of either a 70 degree angled scope or a traditional triangulation with level II or III arthroscopic surgery performed from the endaural and anterior lateral portals are necessary. An important additional concept by him in a condensed version, is that we view the physiological basis of the progressive nature of the joint dysfunction and modeling that occurs in response to adverse mechanical 
loading, and that it is simply unrealistic to believe that we can perform procedures within the joint to reverse the physiologic remodeling that has already occurred, one can only hope to arrest the dysfunction and the resulting degeneration. The goals of treatment should include decreasing functional load and increasing the capacity of cells to accomplish articular remodeling. With mobilization of tissues within and around the joint, combined with reduction of load concentrations, enhanced mesenchymal cell reprogramming may be achieved thereby allowing enhanced potential for ongoing functional remodeling and joint health. (Moses J, 2020, unpublished data).

Although the outcome of lateral eminencia release and capsular stretching without surgical removal of plicae for TMJ lateral impingement reported good results, it cannot discuss regarding the later course of plicae.

\section{Intra-articular impingement/entrapment pathologies}

Impingement/entrapment syndromes are common causes of pain and are well known to affect synovial joints throughout the body. The orthopedic literature has documented the occurrence of impingement syndromes of the knee, shoulder, hip, and other synovial joints. Although synovial plicae are common and usually asymptomatic, there are a variety of etiologic factors that can cause these plicae to develop inflammation, edema, synovial hyperplasia ultimately causing impingement and impaired biomechanical functioning. Arthroscopy of some symptomatic knee joints have confirmed mediopatellar plica syndrome, which is caused by pathologic changes of the mediopatellar plica resulting in patello-femoral impingement (4). Impingement of intra-articular tissues can lead to progression of synovial inflammation, fibrosis decreased elasticity and localized chondromalacia causing a furthering of pain and reduced mobility (5). Similarly, common impingement syndromes involve the subacromial space of the shoulder, femoroacetabular impingement of the hip and anterior impingement of the ankle.

In this review, all reference to the term "synovial entrapment" pertains to an intra-auricular pathologic condition. However, the term "entrapment" in medicine generally refers to a compression of a peripheral nerve or artery usually between ligamentous and bony structures (38). When a nerve is entrapped, the resultant neuropathy that develops causes symptoms of pain, numbness and tingling. Common entrapment entities include carpal tunnel (median nerve entrapment), ulnar nerve entrapment, popliteal artery syndrome, tarsal tunnel syndrome (posterior tibial nerve entrapment) and there are many others. These entrapment syndromes are extra-articular and are not to be confused with impingement syndromes, which are intraarticular. The potential for extra-articular entrapment of the auriculotemporal nerve causing symptoms due to its proximity to the medial aspect of the mandibular condyle has been reported in the literature (39-41). However, extraarticular entrapment syndromes as a cause of significant TMJ symptoms are speculative, considered rare and not included in this discussion.

As a synovial joint, impairment of TMJ function due to impingement should be expected to occur, and has been described by Moses $(14)$ and Kirk et al. $(15,16)$. Synovial plicae are common findings in knee joints $(4,5,42,43)$ and usually asymptomatic. The presence of synovial plicae in the TMJ are well documented $(6,8,9)$. The discovery of a synovial plica during TMJ arthroscopy raises several questions. Are these synovial plicae causing impingement resulting in symptoms? Or, are these normal intraarticular structures that may be incidental findings, similar to findings that are observed in knee joints? Dupont (4) has indicated that in knee arthroscopy, there are no morphologic characteristics that allow assessment of the pathologic aspects of synovial plicae. Furthermore, only histopathology demonstrating inflammatory and/or fibrotic changes and resolution of symptoms with removal of the medial plica can confirm that the medial plica is causing symptoms. Additional pathologic findings seen on knee arthroscopy often make the evaluation of the plica's responsibility in symptoms difficult to analyze. Therefore, the presence of a medial plica along with other pathologic tissues, cannot definitively confirm whether the plica is a major cause of symptoms. Interestingly, the incidence of medial plica syndrome is estimated to be present in $3 \%$ of knee arthroscopies.

The question remains for the oral and maxillofacial surgeon as to how one should treat a synovial plica seen during arthroscopic examination of the TMJ? A logical approach would be to remove the plica and submit the specimen for histopathologic examination (9). Patients undergoing TMJ arthroscopy have limited function and a reduction in the ability to tolerate loads, as well as reduced functional range of motion. Since a major goal of arthroscopic surgery is to remove impediments to mobility, the surgeon should eliminate the potential for a synovial plica to cause impingement, inflammation and reduced mobility. 


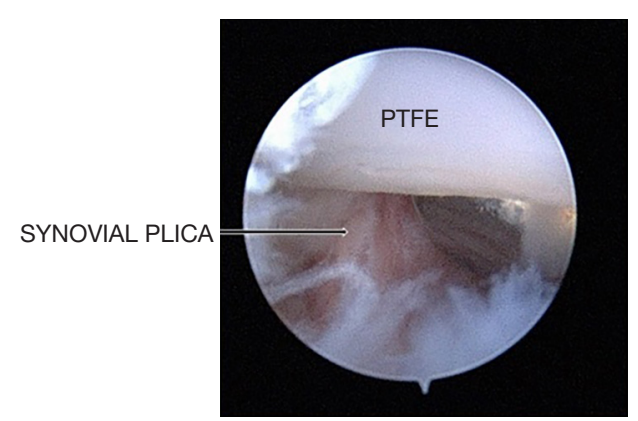

Figure 14 Synovial plica with significant fibrosis present during arthroscopic surgery of a right TMJ total joint prosthesis. (Israel H, 2020, unpublished data).

Symptomatic impingement syndromes have been well documented in patients who have undergone total arthroplasty and joint replacements of the hip (44-47). A recent study on the early results of arthroscopic surgery in symptomatic patients with prior TMJ total joint prosthesis surgery demonstrated favorable outcomes (48). Inflamed synovial plica with fibrosis were seen in some patients (Figure 14, Israel H, 2020, unpublished data) and these were removed with advanced operative arthroscopy techniques. As we attain more experience in this area, we are likely to see the same pathologies that are seen in total joint replacements in other joints.

Arthroscopic studies with synovial fluid analysis have demonstrated that etiologic factors (e.g., joint overload, impaired mobility) cause biochemical alterations and pathologic changes in intra-articular tissues, affecting cartilage and synovium altering structure and impairing function. Thus, synovial inflammation, fibrosis, hypervascularity occurs in damaged intra-articular tissues, and when this occurs in TMJ synovial plicae it is likely to be a cause, but not necessarily the only cause, of symptoms. Surgeons need to be cautious in attributing all symptoms based on the pathologies seen in one type of intra-articular tissue, such as the disc, cartilage, synovium, and plicae, as there is interdependence of the maintenance of structure and function which results in a biomechanically functional and painless joint. Progressive damage and pathology involving one type of intra-articular tissue will ultimately result in changes and pathology in other intra-articular tissues.

\section{Predictions for the future}

The role of synovial plicae has not been clearly defined in TMJ health and disease, although there are numerous reports on synovial plicae of other synovial joints. Synovial plicae of the TMJ are rarely detected, whereas, 4.0 Tesla MR imaging may detect the variety of synovial forms and pathologies including plicae as in other synovial joints. Careful arthroscopic exploration has the ability to detect them, and their removal with histopathologic examination will provide us the immunohistochemical findings in patients with TMJ inflammatory/degenerative diseases. In the treatment of TMJ internal derangement, there has been a paradigm shift from emphasis on disc position to disc mobility. Disc and joint mobility has been accepted as a biomechanical explanation stimulating adaptive joint tissue responses including potential formation of fibrocartilaginous structures. Further biochemical studies of the synovium, including plicae, by immunohistochemical and molecular analysis may clarify the cascade of events leading to inflammatory/degenerative TMJ disorders. The proposed concept of intra-articular synovial entrapment in TMJ-TJR failure patients will lead to breakthroughs in minimally invasive surgical interventions as well as a significant research to identify the origin of synovial entrapment tissues. Early arthroscopic surgical intervention will likely prevent heterotopic bone formation and maximize joint mobility and function.

\section{Acknowledgments}

Funding: None.

\section{Footnote}

Conflicts of Interest: All authors have completed the ICMJE uniform disclosure form (available at https://fomm. amegroups.com/article/view/10.21037/fomm-20-45/coif). The series "Temporomandibular Joint Disorders Diagnosis and Management - What Does the Future Hold?" was commissioned by the editorial office without any funding or sponsorship. Dr. HI reports personal fees from Therapeutic Mobilization Devices (TMD), LLC, outside the submitted work; in addition, Dr. HI has a patent E-Z Flex Passive Motion Jaw Exerciser issued. The authors have no other conflicts of interest to declare.

Ethical Statement: The authors are accountable for all aspects of the work in ensuring that questions related to the accuracy or integrity of any part of the work are appropriately investigated and resolved. 
Open Access Statement: This is an Open Access article distributed in accordance with the Creative Commons Attribution-NonCommercial-NoDerivs 4.0 International License (CC BY-NC-ND 4.0), which permits the noncommercial replication and distribution of the article with the strict proviso that no changes or edits are made and the original work is properly cited (including links to both the formal publication through the relevant DOI and the license). See: https://creativecommons.org/licenses/by-nc-nd/4.0/.

\section{References}

1. Zmerly H, Akkawi I, Citarella R, et al. Clinical management of medial patellar plica syndrome: mini review from diagnosis to treatment. Curr Rheumatol Rev 2020;16:9-11.

2. Wong JS, Lalam R. Plicae: where do they come from and when are they relevant? Semin Musculoskelet Radiol 2019;23:547-68

3. Lee PYF, Nixion A, Chandratreya A, et al. Synovial plica syndrome of the knee: a commonly overlooked cause of anterior knee pain. Surg J (N Y) 2017;3:e9-16.

4. Dupont JYI. Synovial plicae of the knee: controversies and review. Clin Sports Med 1997;16:87-122.

5. Schindler OS. The Sneaky Plica' revisited: morphology, pathophysiology and treatment of synovial plicae of the knee. Knee Surg Sports Traumatol Arthrosc 2014;22:247-62.

6. Murakami K, Hoshino K. Regional anatomical nomenclature and arthroscopic terminology in human temporomandibular joints. Okajimas Folia Anat Jpn 1982;58:745-60.

7. Heffez L, Blaustein D. Diagnostic arthroscopy of the temporomandibular joint. Part I: normal arthroscopic findings. Oral Surg Oral Med Oral Pathol 1987;64:653-70.

8. Murakami K, Hori S, Yamaguchi Y, et al. Synovial plicae and temporomandibular joint disorders: surgical findings. J Oral Maxillofac Surg 2015;73:827-33.

9. Hakim MA, Christensen B, Ahn DY, et al. Correlation of arthroscopic and histologic findings in synovial membrane disease of the temporomandibular joint. J Oral Maxillofac Surg 2019;77:e48-50.

10. Furstman L. Embryology. In: Sarnat BG, Laskin DM. The temporomandibular joint. 3rd ed. Springfield: Charles Thomas Pub, 1980:52-69.

11. Geraghty RM, Spear M. Evidence for plical support of the patella. J Anat 2017;231:698-707.

12. Murakami K, Hoshino K. Histological studies on the inner surface of the articular cavities of human temporomandibular joints with special reference to arthroscopic observations. Anat Anz 1985;160:167-77.

13. Ohmura Y. Histological observations on the structure of the lateral wall of the human temporomandibular joint. Kokubyo Gakkai Zasshi 1984;51:465-92.

14. Moses JJ. Lateral impingement syndrome and endaural surgical technique. Oral Maxillofac Surg Nor Amer 1989;1:165-83.

15. Kirk WS Jr, Kirk BS. A biomechanical basis for primary arthroplasty of the temporomandibular joint. Oral Maxillofac Surg Clin North Am 2006;18:345-68, vi.

16. Kirk WS Jr. Lateral impingements of the temporomandibular joint: a classification system and MRI imaging characteristics. Int J Oral Maxillofac Surg 2013;42:223-8.

17. Kirk WS Jr. Illustrated surgical techniques for management of impingements of the temporomandibular joint. Int J Oral Maxillofac Surg 2013;42:229-36.

18. Watanabe M, Takeda S, Ikeuchi H. Atlas of arthroscopy. 3rd ed. Tokyo: Igaku-Shoin, 1978.

19. He R, Yang L, Chen G, et al. Substance-P in symptomatic mediopatellar plica as a predictor of patellofemoral pain. Biomed Rep 2016;4:21-6.

20. Kopp S. Neuroendocrine, immune, and local responses related to temporomandibular disorders. J Orofac Pain 2001;15:9-28.

21. Yoshida H, Fujita S, Nishida S, et al. The expression of substance $\mathrm{p}$ in human temporomandibular joint samples: an immunohistochemical study. J Oral Rehabil 1999;26:338-44.

22. Sato J, Segami N, Yoshitake Y, et al. Specific expression of substance $\mathrm{P}$ in synovial tissues of patients with symptomatic, non-reducing internal derangement of the temporomandibular joint: Comparison with clinical findings. Br J Oral Maxillofac Surg 2007;45:372-7.

23. Yang CC, Lin CY, Wang HS, et al. Matrix metalloproteases and tissue inhibitors of metalloproteinases in medial plica and pannus-like tissue contribute to knee osteoarthritis progression. PLoS One 2013;8:e79662.

24. Murakami K. Rationale of arthroscopic surgery of the temporomandibular joint. J Oral Biol Craniofac Res 2013;3:126-34.

25. Israel HA. Internal derangement of the temporomandibular joint: new perspectives on an old problem. Oral Maxillofac Surg Clin North Am 2016;28:313-33.

26. Kubota $\mathrm{E}$, Imamura $\mathrm{H}$, Kubota $\mathrm{T}$, et al. Interleukin 1beta and omelysin (MMP3) activity of synovial fluid as possible markers of osteoarthritis in the temporomandibular joint. J 
Oral Maxillofac Surg 1997;55:20-7.

27. Kellesarian SV, Al-Kheraif AA, Vohra F, et al. Cytokine profile in the synovial fluid of patients with temporomandibular joint disorders: a systematic review. Cytokine 2016;77:98-106.

28. Ibi M. Inflammation and temporomandibular joint derangement. Biol Pharm Bull 2019;42:538-42.

29. Loreto C, Filetti V, Almeida LE, et al. MMP-7 and MMP9 are overexpressed in the synovial tissue from severe temporomandibular joint dysfunction. Eur J Histochem 2020;64:3113-24.

30. Behzadi F, Mandell JC, Smith SE, et al. Temporomandibular joint imaging: current clinical applications, biochemical comparison with the intervertebral disc and knee meniscus, and opportunities for advancement. Skeletal Radiol 2020;49:1183-93.

31. Moses JJ, Poker ID. TMJ arthroscopic surgery: an analysis of 237 patients. J Oral Maxillofac Surg 1989;47:790-4.

32. Moses JJ, Topper DC. A functional approach to the treatment of temporomandibular joint internal derangement. J Craniomandib Disord 1991;5:19-27.

33. Moses JJ, Sartoris D, Glass R, et al. The effect of arthroscopic surgical lysis and lavage of the superior joint space on TMJ disc position and mobility. J Oral Maxillofac Surg 1989;47:674-8.

34. Moses JJ, Lo HH. Tomographic changes in the temporomandibular joint following arthroscopic surgery with lysis and lavage and eminentia release J Orofac Pain 1994;8:407-12.

35. Moses JJ. Articular pathology: disc displacement and Lateral impingement syndrome. In: Thomas M, Bronstein SL. Arthroscopy of the temporomandibular joint. Philadelphia: WB Saunders Co., 1991:249-57.

36. Moses JJ, Poker I. Temporomandibular joint arthroscopy: the endaural approach. Int J Oral Maxillofac Surg 1989; 18:347-51.

37. Moses JJ. Endaural arthroscopic approach. In: Thomas M, Bronstein SL. Arthroscopy of the temporomandibular joint. Philadelphia: WB Saunders Co., 1991:192-8.

38. Dong Q, Jacobson JA, Jamadar DA, et al. Entrapment

doi: $10.21037 /$ fomm-20-45

Cite this article as: Murakami K, Moses JJ, Israel H, McCain JP. Synovial plica, lateral impingement and intraarticular impingement/entrapment pathologies of the temporomandibular joint. Front Oral Maxillofac Med 2020;2:24. neuropathies in the upper and lower limbs: anatomy and MRI features. Radiol Res Pract 2012;2012:230679.

39. Karl HW, Trescot AM. Nerve entrapment headaches at the temple: Zygomaticotemporal and/or auriculotemporal nerve? Pain Physician 2019;22:E15-36.

40. Fernandes PRB, de Vasconsellos HA, Okeson JP, et al. The anatomical relationship between the position of the auriculotemporal nerve and mandibular condyle. Cranio 2003;21:165-71.

41. Johansson AS, Isberg A, Isacsson G. A radiographic and histologic study of the topographic relations in the temporomandibular joint region: Implications for a nerve entrapment mechanism. J Oral Maxillofac Surg 1990;48:953-61.

42. Ogata S, Uhthoff HK. The development of synovial plicae in human knee joints: an embryologic study. Arthroscopy 1990;6:315-21.

43. Patel D. Plica as a cause of anterior knee pain. Orthop Clin North Am 1986;17:273-77.

44. Lachiewicz PF, Kauk JR. Anterior iliopsoas impingement and tendinitis after total hip arthroplasty. J Am Acad Orthop Surg 2009;17:337-44.

45. Dora C, Houweling M, Koch P, et al. Iliopsoas impingement after total hip replacement: the results of non-operative management, tenotomy or acetabular revision. J Bone Joint Surg Br 2007;89:1031-5.

46. Chalmers BP, Sculco PK, Sierra RJ, et al. Iliopsoas impingement after primary total hip arthroplasty: operative and nonoperative treatment outcomes. J Bone Joint Surg Am 2017;99:557-64.

47. Guicherd W, Bonin N, Gicquel T, et al. Endoscopic or arthroscopic iliopsoas tenotomy for iliopsoas impingement following total hip replacement. A prospective multicenter 64-case series. Orthop Traumatol Surg Res 2017;103:S207-14.

48. Davis CM, Hakim M, Choi DD, et al. Early clinical outcomes of arthroscopic management of the failing alloplastic temporomandibular joint prosthesis. J Oral Maxillofac Surg 2020;78:903-7. 